\title{
Cytomegalovirus pneumonitis following temozolomide for the treatment of glioblastoma multiforme
}

\author{
Salma Al Bahrani and Coleman Rotstein* \\ Division of Infectious Diseases, Department of Medicine, University of Toronto, University Health Network, Toronto, Ontario, Canada
}

\begin{abstract}
Temozolomide (TMZ) is considered to be standard chemotherapy for the treatment of glioblastoma, but may cause lymphocytopenia thus potentiating the risk for opportunistic infections. Although one might anticipate that recurrent CMV infection may be precipitated by temozolomide immunosuppression, few cases of TMZ-induced cytomegalovirus (CMV) recurrent infection have been reported. Herein, we report a patient with malignant glioma who developed CMV pneumonia following TMZ therapy. The patient developed significant recurrent CMV infection with pneumonitis diagnosed by PCR (DNA analysis). Antiviral treatment with intravenous ganciclovir was administered. For patients with prior CMV exposure, it would be prudent to monitor CMV viral loads periodically to avoid recurrent CMV infection after exposure to TMZ and corticosteroid chemotherapy.
\end{abstract}

\section{Introduction}

Temozolomide (TMZ) is an oral alkylating agent with antitumor activity approved for the treatment of newly diagnosed glioblastoma multiforme and refractory anaplastic astrocytoma $[1,2]$. The combined use of TMZ chemotherapy with radiotherapy after surgical resection has resulted in a statistically significant survival benefit for up to five years of follow up, compared with radiotherapy alone for the treatment of glioblastoma [3,4]. However, TMZ use is not without potential harmful effects. Myelotoxicity related to TMZ has been reported in 3.5$7 \%$ of patients [4-6]. Due to its production of lymphocytopenia, TMZ is thought to promote opportunistic infection, due to Pneumocystis jirovecii as well as other pathogens such as cytomegalovirus (CMV). Concurrent steroid usage may also enhance the risk for these infections. Prophylaxis against Pneumocystis jirovecii pneumonia is routinely advised by the United States Food and Drug Administration and is to be continued until normalization of the lymphocyte count has been achieved. Herein, we report a case of CMV infection that occurred in patient treated with TMZ therapy.

\section{Case report}

A 52 year old male was admitted to the hospital with worsening dyspnea on exertion of one week. He had previously been diagnosed with a grade IV glioblastoma tumor (World Health Organization Classification 2000). Treatment for the malignancy consisted of radiotherapy and concomitant TMZ (75 $\mathrm{mg} / \mathrm{kg}$ per day) after surgical resection of his tumor. He received only ten capsules of TMZ before it was stopped because of elevated liver enzymes (aspartate aminotransferase 609 Units/L and alanine aminotransferase 1658 Units/L). He had also been taking dexamethasone $6 \mathrm{mg} /$ day to reduce tumor-induced cerebral edema for at least 3 weeks before his admission to the hospital. He had not been prescribed prophylactic trimethoprimsulfamethoxazole. Complete blood count monitoring was undertaken while undergoing his chemotherapy treatment and demonstrated a consistent lymphocytopenia (consistently $<0.60 \times 10^{9} / \mathrm{L}$ ).

At the time of his presentation to the hospital, he complained of dyspnea, fatigue, and a dry cough, but no fever. Upon physical examination, his blood pressure was $110 / 60 \mathrm{~mm} \mathrm{Hg}$, pulse 85 beats/minute and the respiratory rate was 25 breaths/minute. On auscultation, the patient displayed respiratory crackles throughout both lung fields posteriorly. His complete blood count revealed leukopenia and thrombocytopenia with an absolute lymphocyte count of $0.0 \times 10^{9} / \mathrm{L}$. No abnormality was detected in his serum electrolytes except for hyponatremia related to syndrome of inappropriate antidiuretic hormone and elevated liver enzymes thought to be druginduced secondary to TMZ. With oxygen supplementation of $4 \mathrm{~L} /$ minute via nasal prongs, his oxygen saturation was $89 \%$.

After hospitalization, piperacillin-tazobactam and azithromycin were administered intravenously. A high resolution CT scan of the chest was performed, and extensive ground glass opacities with bilateral nodules were detected. No pulmonary emboli were observed. Due to his low arterial oxygen saturation, bronchoscopic examination could not be undertaken. Blood samples were taken for viral detection by PCR testing. Bacterial culture of the sputum and sputum for a Pneumocystis jirovecii stain were also ordered. Stains for Pneumocystis jirovecii were negative. In addition, a urine sample for Legionella antigen was also negative. After a few days of no clinical improvement and deterioration in his respiratory status, CMV DNA PCR was performed and this detected 677,000 international units (IU)/mL. Ganciclovir intravenous therapy $(5 \mathrm{mg} / \mathrm{kg}$ every 12 hours) was immunoglobulin therapy intravenously as well.

The patient's condition however, failed to improve and he was transferred to the intensive care unit where high flow oxygen therapy was required. Despite these measures, his CMV PCR continued to be

Correspondence to: Coleman Rotstein, MD, Division of Infectious Diseases, Department of Medicine, University of Toronto, University Health Network, 200 Elizabeth Street, PMB 11-139, Toronto, ON, M5G 2C4, Canada, Tel: 4163404800/6663; Fax: 4163405442; E-mail: Coleman.Rotstein@uhn.ca

Key words: Temozolomide CMV gliobastoma

Received: July 16, 2017; Accepted: August 11, 2017; Published: August 14, 2017 
elevated $(2,770,000 \mathrm{IU} / \mathrm{mL})$. Because of his diminishing pulmonary status with concomitant rising CMV PCR, further chemotherapy was deferred and he succumbed due to his CMV infection and progressive tumor.

\section{Discussion}

TMZ, a novel alkylating agent, used in the treatment of glioblastoma multiforme and recurrent anaplastic astrocytoma, has also been employed for malignant melanomas, metastatic neuroendocrine tumors and some brain metastases. It is generally well-tolerated although its main toxicities are nausea, vomiting, anorexia and thrombocytopenia. Leukopenia, a less common complication noted with TMZ use, has been reported in 7\% of patients [4]. Moreover, TMZ produces $\mathrm{CD} 4+\mathrm{T}$-cell dysfunction [5,6]. In patients with malignant gliomas, peritumoral edema is also a complication. Corticosteroids are the therapeutic mainstay to prevent this edema. However, corticosteroids also contribute to the immunocompromised state by decreasing the peripheral lymphocyte count. Selective CD4 lymphocyte depletion may cause an increased susceptibility to opportunistic infections including varicella-zoster virus, CMV and Pneumocystis jirovecii. Thus, it is not surprising that Pneumocystis jirovecii infection associated with TMZ treatment has been reported [5]. Therefore, the Canadian Glioblastoma Multiforme Recommendations Committee has also advocated that prophylaxis for Pneumocystis jirovecii be considered when administering TMZ [7]. Other investigators have advocated that PCP prophylaxis should be recommended only if lymphocytopenia occurs [8].

In addition, there has been a report of two cases of CMV infection associated with TMZ [9]. In this report, 4 additional cases of CMV infection were culled from the literature and 5 of the 6 cases involved TMZ administration with corticosteroids with evidence of CMV pneumonitis in 3 patients. Although our patient had a grade IV astrocytoma, TMZ was given per Stupp's protocol [2] and followed Eberlein et al's practice where patients with grade III astrocytoma had an increased chance of survival if treated with TMZ [8]. As his CMV recurrent infction progressed in the hospital, our patient developed significant fever, hypoxia and radiological pulmonary infiltrates with a highly elevated blood CMV PCR in keeping with CMV infection involving the lungs $[10,11]$. But, a diagnostic bronchoscopic examination could not be performed due to severe hypoxia. However, based on the aforementioned clinical criteria, the diagnosis of symptomatic CMV recurrent infection was made and so intravenous ganciclovir therapy was initiated. Taken together, these data emphasize that patients treated with TMZ and receiving concomitant steroids may also be at risk of CMV infection reactivation. Thus, one should be cognizant of the risk for recurrent CMV infection when employing TMZ chemotherapy in combination with corticosteroids in individuals who have had prior CMV exposure (evidence of positive CMV antibody). Subsequently pre-emptive periodic monitoring of CMV viral loads in such patients would be prudent..

In conclusion, patients receiving TMZ treatment must be evaluated closely for myelosuppression and specifically for lymphocytopenia. Clinicians must be cognizant of the possible opportunistic infections that may occur during TMZ treatment with concomitant radiotherapy and corticosteroid usage that include Pneumocystis jirovecii for which prophylaxis should be administered and CMV. Pre-emptive periodic CMV viral load monitoring should therefore be conducted if prior evidence of CMV infection is present to anticipate the development of symptomatic CMV infection because of this predisposition to opportunistic infection.

\section{References}

1. Newlands ES, Stevens MF, Wedge SR, Wheelhouse RT, Brock C (1997) Temozolomide: a review of its discovery, chemical properties, pre-clinical development and clinical trials. Cancer Treat Rev 23: 35-61. [Crossref]

2. Stupp R, Mason WP, van den Bent MJ, Weller M, Fisher B, et al. (2005) Radiotherapy plus concomitant and adjuvant temozolomide for glioblastoma. N Engl J Med 352: 987 996. [Crossref]

3. Athanassiou H, Synodinou M, Maragoudakis E, Paraskevaidis M, Verigos C, et al (2005) Randomized phase II study of temozolomide and radiotherapy compared with radiotherapy alone in newly diagnosed glioblastoma multiforme. J Clin Oncol 23(10): 2372-2377. [Crossref]

4. Cohen MH, Johnson JR, Pazdur R (2005) Food and Drug Administration Drug approval summary: temozolomide plus radiation therapy for the treatment of newly diagnosed glioblastoma multiforme. Clin Cancer Res 11(19 Pt 1): 6767-6771. [Crossref]

5. Schwarzberg AB, Stover EH, Sengupta T, Michelini A, Vincitore M, et al. (2007) Selective lymphopenia and opportunistic infections in neuroendocrine tumor patients receiving temozolomide. Cancer Invest 25(4): 249-255. [Crossref]

6. Su YB, Sohn S, Krown SE, Livingston PO, Wolchok JD, et al. (2004) Selective CD4+ lymphopenia in melanoma patients treated with temozolomide: a toxicity with therapeutic implications. J Clin Oncol 22: 610-616. [Crossref]

7. Mason WP, Maestro RD, Eisenstat D, Forsyth P, Fulton D, et al. (2007) Canadian recommendations for the treatment of glioblastoma multiforme. Curr Oncol 14: 110117. [Crossref]

8. Eberlein KH, Nagel B, Franz K, Imhoff D, Seifert V, et al. (2006) Concomitant radiochemotherapy with temozolomide in non-selected patients with newly diagnosed high-grade gliomas. Anticancer Res 26(6c): 4959-4964. [Crossref]

9. Meije Y, Lizasoain M, García-Reyne A, Martínez P, Rodríguez V, et al. (2009) Emergence of cytomegalovirus disease in patients receiving temozolomide: report of two cases and literature review. Clin Infect Dis 50(12): e73-e76. [Crossref]

10. Wolf DG, Spector SA (1993) Early diagnosis of human cytomegalovirus disease in transplant recipients by DNA amplification in plasma. Transplantation 56(2): 330-334. [Crossref]

11. Ljungman P, de la Camara R, Cordonnier C, Einsele H, Engelhard D, et al. (2008) Management of CMV, HHV-6, HHV-7 and Kaposi-sarcoma herpesvirus (HHV-8) infections in patients with hematological malignancies and after SCT. Bone Marrow Transplant 42(4): 227-240. [Crossref]

Copyright: (C2017 Bahrani SA. This is an open-access article distributed under the terms of the Creative Commons Attribution License, which permits unrestricted use, distribution, and reproduction in any medium, provided the original author and source are credited. 